\title{
二氟烯醇硅醚与烯烃的马氏区域选择性氢二氟烷基化反应
}

\author{
陈国术刘运林* \\ (广州大学化学化工学院 广州 510006)
}

\section{Regioselective Markovnikov Hydrodifluoroalkylation of Difluoroenoxysilanes with Alkenes}

\author{
Chen, Guoshu \\ Liu, Yunlin* \\ (School of Chemistry and Chemical Engineering, Guangzhou University, Guangzhou 510006)
}

烯烃是一类广泛应用于工业生产和实验室合成的 重要基础化工原料. 通过烯烃的氢二氟烷基化反应, 可 在烯烃中一步选择性地引入二氟烷基结构单元, 实现一 些高附加值的复杂功能分子的合成, 因而引起了合成和 药物化学工作者的广泛关注 ${ }^{[1]}$.

近年来, 通过光氧化还原催化或外加氧化剂的方 法, 经过自由基历程已成功实现了烯烃的反马氏区域选 择性氢二氟烷基化反应合成直链型的加成产物(Scheme $1, a)^{[2]}$. 尽管该领域已取得了一定进展, 但这些方法仍 然具有一定的局限性: 一方面, 为了生成稳定的自由基 加成中间体, 导致其通常只能专一性地发生反马氏区域 选择性加成, 而无法通过马氏区域选择性加成获得支链
型产物; 另一方面, 烯烃的底物范围局限性很大, 绝大 部分只适用于单烷基取代的烯烃, 多取代烯烃或芳基烯 烃往往并不适用. 因此, 探索新的反应机理、发展简单 实用的烯烃马氏区域选择性氢二氟烷基化方法仍然是 该领域的一个巨大挑战.

近年来, 二氟烯醇硅醚被广泛用作偕二氟碳负离子 的前体，已经成为构建偕二氟烷基化合物的强有力合成 子. 在这一领域, 卿风翎 ${ }^{[3]}$ 、施敏 ${ }^{[4]}$ 、周剑 ${ }^{[5]}$ 、马军安 ${ }^{[6]}$ 、 张新刚 ${ }^{[7]}$ 和彭勃 ${ }^{[8]}$ 等课题组发展了系列二氟烯醇硅醚参 与的二氟烷基化反应. 基于对二氟烯醇硅醚性质的认 识, 华东师范大学化学与分子工程学院周剑和余金生等 考虑是否能利用酸活化烯烃产生碳正离子中间体, 并使

(a) anti-Markovnikov alkene hydrodifluoroalkylation based on radical processes (previous work)

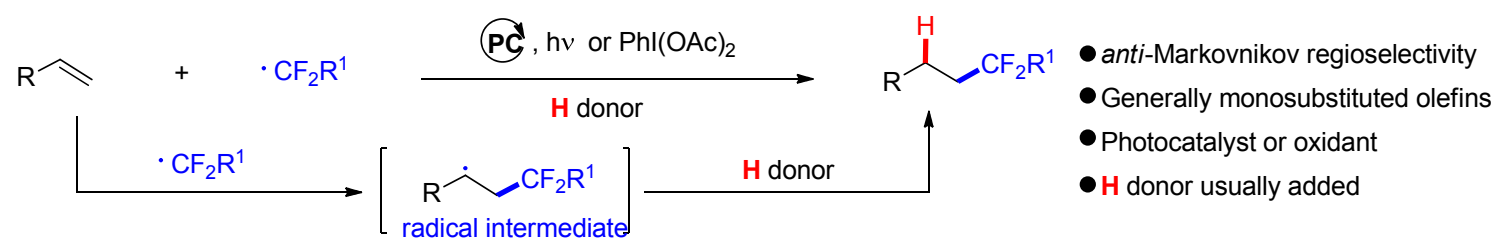

(b) Unprecedented Markovnikov alkene hydrodifluoroalkylation (this work)

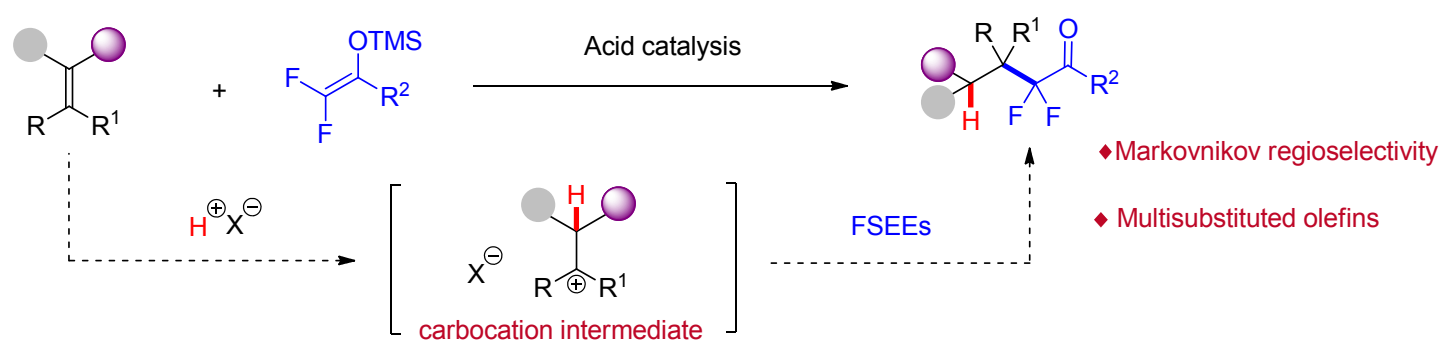

图式 1 烯烃的氢二氟烷基化研究现状及反应设计

Scheme 1 State of the art and present reaction design

* Corresponding author. E-mail: ylliu@gzhu.edu.cn. Published online January 29, 2021. 
用二氟烯醇硅醚作为亲核试剂来实现烯烃的马氏区域 选择性氢二氟烷基化(Scheme 1, b).

近日，该团队结合布朗斯特酸以及金属路易斯酸催 化, 成功发展了六水合高氯酸镁催化的烯烃与二氟烯醇 硅醚的马氏区域选择性氢二氟烷基化反应 ${ }^{[9]}$. 研究发现, 该反应条件温和，官能团兼容性强，底物普适性极广， 不仅适用于各种单取代、二取代、三取代、甚至四取代 的烯烃, 而且还能通过后期氢二氟烷基化修饰在天然产 物与药物分子骨架中, 引入 $\alpha, \alpha$-二氟代烷基酮结构单元. 此外，该方法不仅能实现产物的克级规模制备，而且产 物能进行多种衍生化合成结构丰富的高附加值氟代烷 基化合物(Scheme 2). 进一步的生物活性测试研究表明, 所合成的二氟烷基酮类化合物具有较强的抗人结直肠 癌细胞活性, 因此该类含氟化合物在抗肿瘤药物开发中 具有潜在的应用价值.
机理实验研究表明，催化量的非螯合性有机碱 2,6二叔丁基吡啶的加入完全抑制该反应; 高氯酸也能催化 该反应，但产率略有下降. 这些结果说明 $\mathrm{Mg}\left(\mathrm{ClO}_{4}\right)_{2} \bullet$ $6 \mathrm{H}_{2} \mathrm{O}$ 可能是作为潜布朗斯特酸(hidden Brønsted acid $)^{[10]}$ 缓慢释放出高氯酸, 避免因酸浓度过高导致烯烃聚合, 从而比直接使用高氯酸催化取得更高的产率. 氝代实验 表明产物的质子主要来自于高氯酸镁水合物中的结晶 水. 使用叔醇作为底物, 发现反应除了能获得相应的目 标产物，还伴随着反式烯烃的生成，进一步说明反应可 能是经历碳正离子的历程.

利用二氟烯醇硅醚实现的烯烃的马氏区域选择性 氢二氟烷基化反应，为构建系列高附加值的 $\beta$-季(叔) 碳- $\alpha, \alpha$-二氟烷基酮类化合物提供了一种高效、温和的新 方法，同时也为烯烃的氢氟烷基化反应提供了新思路.
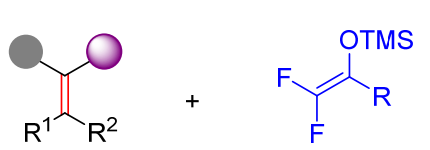

$\mathrm{Mg}\left(\mathrm{ClO}_{4}\right)_{2} \cdot 6 \mathrm{H}_{2} \mathrm{O}$

(3 $10 \mathrm{~mol} \%)$

$\mathrm{ClCH}_{2} \mathrm{CH}_{2} \mathrm{Cl}$, r.t., in air
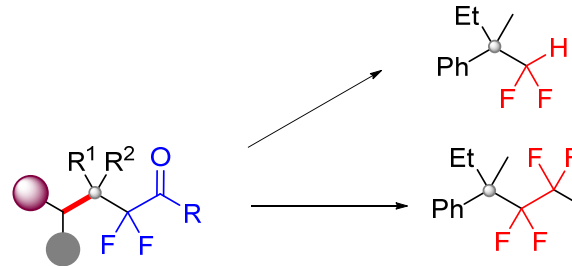

71 examples, up to $99 \%$ yield

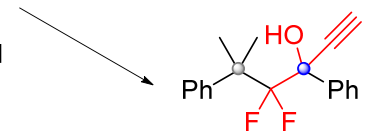

Representative examples of late-stage diversification

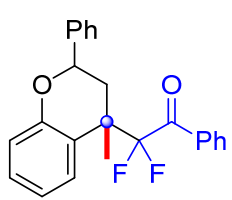

Flavononid derivative<smiles>CC(=O)CCC(C)(c1ccc(-c2ccccc2)cc1)C(F)(F)C(=O)c1ccccc1</smiles>

Fenbufen derivative

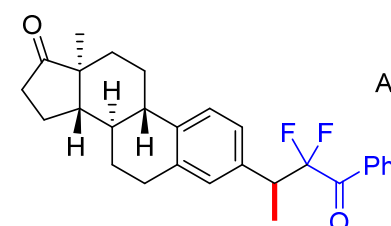

Estrone derivative

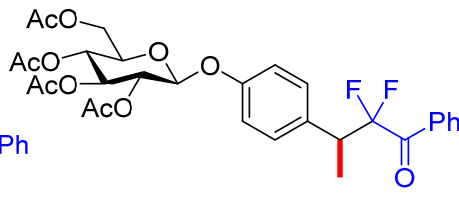

Glucose derivative

图式 $2 \mathrm{Mg}\left(\mathrm{ClO}_{4}\right)_{2} \cdot 6 \mathrm{H}_{2} \mathrm{O}$ 催化的二氟烯醇硅醚与烯烃的马氏区域选择性氢二氟烷基化反应

Scheme $2 \mathrm{Mg}\left(\mathrm{ClO}_{4}\right)_{2} \cdot 6 \mathrm{H}_{2} \mathrm{O}$ catalyzed Markovnikov hydrodifluoroalkylation of difluoroenoxysilanes with allkenes

\section{References}

[1] Kirsch, P. Modern Fluoroorganic Chemistry: Synthesis, Reactivity and Applications, 2nd ed., Wiley-VCH, Weinheim, 2013

[2] For a review, see: (a) Huang, H.; Wang, X.; Wang, J. Chin. J. Org Chem. 2019, 39, 1 (in Chinese).

(黄航, 王兮, 王剑波, 有机化学, 2019, 39, 1.)

For selected examples, see: (b) Ma, G.; Wan, W.; Li, J.; Hu, Q.; Jiang, H.; Zhu, S.; Wang, J.; Hao, J. Chem. Commun. 2014, 50, 9749.

(c) Lin, Q.-Y.; Xu, X.-H.; Zhang, K.; Qing, F.-L. Angew. Chem., Int. Ed. 2016, 55, 1479 .

(d) Wang, H.; Jui, N. T. J. Am. Chem. Soc. 2018, 140, 163.

(e) Meyer, C. F.; Hell, S. M.; Misale, A.; Trabanco, A. A.; Gouver- neur, V. Angew. Chem., Int. Ed. 2019, 58, 8829.

[3] Chu, L.; Zhang, X.; Qing, F.-L. Org. Lett. 2009, 11, 2197.

[4] Yuan, Z.; Wei, Y.; Shi, M. Chin. J. Chem. 2010, $28,1709$.

[5] Hu, X.-S.; Yu, J.-S.; Zhou, J. Chem. Commun. 2019, 55, 13638.

[6] Li, J.-S.; Liu, Y.-J.; Zhang, G.-W.; Ma, J.-A. Org. Lett. 2017, 19, 6364.

[7] Gao, X.; Cheng, R.; Xiao, Y.-L.; Wan, X.-L.; Zhang, X. Chem. 2019, 5, 2987.

[8] Huang, X.; Zhang, Y.; Zhang, C.; Zhang, L.; Xu, Y.; Kong, L.; Wang, Z.-X.; Peng, B. Angew. Chem., Int. Ed. 2019, 58, 5956.

[9] Hu, X.-S.; He, J.-X.; Dong, S.-Z.; Zhao, Q.-H.; Yu, J.-S.; Zhou, J. Nat. Commun. 2020, 11, 5500 .

[10] Wabnitz, T. C.; Yu, J.-Q.; Spencer, J. B. Chem.-Eur. J. 2004, 10, 484.

(Zhao, C.) 